\title{
PROPERTIES OF CONTINUOUS EXPANSIONS
}

\author{
CARLOS R. BORGES
}

ABSTRACT. We study the properties of continuous expansions, in comparison with contractions. We obtain a few applications which demonstrate the usefulness of expansions.

Given that the inverse of an expansion is a contraction, one is naturally led to the study of expansions by analyzing the behavior of their inverses. It turns out that, in Euclidean spaces, the behavior of expansions and contractions is about the same. But the same cannot be said for Hilbert spaces.

1. Preliminaries. As is customary, we will say that a function $f:$ $(X, d) \rightarrow(Y, \rho)$ is a $\beta$-expansion if $\beta>1$ and $\rho(f(x), f(w)) \geq \beta d(x, w)$. For convenience, we will say that $f$ is a $\gamma$-enlarger if $\gamma>0$ and $\rho(f(x), f(w)) \geq$ $\gamma d(x, y)$. Also, for any function $f: X \rightarrow X$, we let $P=\bigcap_{n=1}^{\infty} f^{n}(X)$.

Note that, unlike contractions, expansions are not necessarily continuous. For example, let $f: E^{1} \rightarrow E^{1}$ be defined by

$$
f(x)= \begin{cases}2 x & \text { for } x \leq 0, \\ 2 x+1 & \text { for } x>0 .\end{cases}
$$

It follows that $f$ is a noncontinuous 2-expansion. (Of course, $f$ is not onto.)

Lemma 1.1. Let $(X, d)$ be a complete metric space, $(Y, \rho)$ any metric space and $f: X \rightarrow Y$ any continuous $\gamma$-enlarger. If $Z$ is a complete subspace of $X$ then $W=f(Z)$ is a complete subspace of $Y$. In particular, $f$ : $X \rightarrow f(X)$ is a bomeomorphism and $f(X)$ is a closed subset of $Y$.

Proof. Clearly $f$ is one-to-one and $d\left(f^{-1}(x), f^{-1}(y)\right) \leq \rho(x, y) / \gamma$ for each $x, y \in f(X)$. It follows that if $\left\{y_{n}\right\}$ is a Cauchy sequence in $W$,

Received by the editors January 2, 1974 and, in revised form, April 12, 1974. AMS (MOS) subject classifications (1970). Primary 54E40; Secondary 54E50. Key words and phrases. Expansions, enlargers, contractions, Brouwer property. 
then $\left\{f^{-1}\left(y_{n}\right)\right\}$ is a Cauchy sequence in $Z$. Therefore, if $\lim _{n} f^{-1}\left(y_{n}\right)=x$ then $\lim _{n}{ }_{n}=f(x)$, since $f$ is continuous. This shows that the image, under $f$, of a complete subspace of $X$ is a complete subspace of $Y$. Since a subspace $S$ of a metric space $M$ is complete implies that $S$ is a closed subset of $M$, it follows that $f$ is a closed continuous function and, hence, a homeomorphism. Furthermore, $f(X)$ is a closed subspace of $Y$.

Lemma 1.2. Let $X$ be any space and $f: X \rightarrow X$ any one-to-one function. Then $f(P)=P$.

Proof. $f(P) \subset P$ : Immediate, since

$$
f(P)=f\left(\bigcap f^{n}(X)\right) \subset \bigcap f f^{n}(X)=\bigcap f^{n+1}(X)=P,
$$

because $f^{n}(X) \supset f^{n+1}(X)$ for each $n$.

$P \subset f(P):$ Let $p \in P$. Then there exists $x_{n} \in X$ such that $f^{n}\left(x_{n}\right)=p$ for $n=1,2, \ldots$. Because $f$ is one-to-one, by induction, we get that $x_{1}=$ $f\left(x_{2}\right)=f^{2}\left(x_{3}\right)=\cdots=f^{n-1}\left(x_{n}\right)=\cdots$. Therefore $x_{1} \in P$ with $f\left(x_{1}\right)=p$, which completes the proof.

Lemma 1.3。Let $(X, d)$ be any complete metric space and $f: X \rightarrow X$ a continuous $\gamma$-enlarger. Then $P$ is a closed (complete) subspace of $X$ and $f(P)=P$.

Proof. From Lemma 1.1 and induction, we get that each $f^{n}(X)$ is a closed subset of $X$. Therefore, we get that $P$ is a closed (hence complete) subspace of $X$. Since $f$ is clearly one-to-one, from Lemma 1.2, we get that $f(P)=P$.

\section{Main results.}

Theorem 2.1. Let $(X, d)$ be any complete metric space and $f: X \rightarrow X$ any continuous $\beta$-expansion. If $P \neq \varnothing$, then $f$ bas a unique fixed point.

Proof. By Lemma 1.3, $P$ is complete. Since $f^{-1}: P \rightarrow P$ is a $1 / \beta$-contraction, $f^{-1}$ has a unique fixed point; therefore, so does $f: X \rightarrow X$.

The bypothesis that $P \neq \varnothing$ is not superfluous, as the following folklore example shows: Let $l_{2}$ be the Hilbert space (of square-summable sequences) and let $f: l_{2} \rightarrow l_{2}$ be defined by

$$
f\left(x_{1}, x_{2}, \cdots\right)=2\left(1-\|\bar{x}\|, x_{1}, x_{2}, \cdots\right),
$$

for each $\bar{x}=\left(x_{1}, x_{2}, \ldots\right) \in l_{2}$. It follows that $f$ is a 2-expansion. Further- 
more, $f(\bar{x}) \neq \bar{x}$ for each $\bar{x} \in l_{2}$. (If $\bar{x}=f(\bar{x})$, then we get that $x_{n+1}=$ $2^{n} x_{1}$ for $n=1,2, \ldots$. This forces $0=x_{1}=x_{2}=\ldots$, but $f(\overline{0}) \neq \overline{0}$.) Clearly $f$ is not onto (for example, $\overline{0} \notin f\left(l_{2}\right)$ ) and $f$ has no fixed points.

Nonetheless, there exists a large class of spaces, including all Euclidean spaces, for which each $\gamma$-enlarger is surjective.

Theorem 2.2. Let $(X, d)$ be any connected, complete metric space with the Brouwer property (i.e., a bomeomorphism in $X$ of an open subset of $X$ is an open subset of $X$ ) and let $f: X \rightarrow X$. be any continuous $\gamma$-enlarger. Then $f$ is an onto bomeomorphism.

Proof. By Lemma 1.1 and hypothesis, $f(X)$ is a clopen subset of $X$. Therefore, since $X$ is connected, $f(X)=X$. Clearly $f^{-1}$ is continuous and thus $f$ is a homeomorphism of $X$ onto $X$.

The following two results are immediate consequences of Theorems 2.1 and 2.2, given that it is well known that every Euclidean space has the Brouwer property.

Corollary 2.3. Let $(X, d)$ be any connected, complete metric space with the Brouwer property. Any $\beta$-expansion $f: X \rightarrow X$ bas a unique fixed point.

Corollary 2.4. Let $X$ be any Euclidean space. Then any $\beta$-expansion $f: X \rightarrow X$ bas a unique fixed point.

The hypotheses of the preceding corollaries are definitely not superfluous, as the following trivial example shows: Let $f:[1,+\infty[\rightarrow[1,+\infty[$ be defined by $f(x)=2 x$. Then $f$ is a 2-expansion without fixed points.

\section{Applications.}

Proposition 3.1. Let $f: E^{n} \rightarrow E^{n}$ be continuous and satisfy the condition $|f(x)-f(y)| \geq K|x-y|, K>1$. Then $f$ bas a unique fixed point.

Proof. Immediate from Corollary 2.4.

Proposition 3.2. Let $(G, d)$ be a connected, complete metric group and $b: G \rightarrow G$ a $\gamma$-enlarger, continuous bomomorphism. Then either $b$ is $a$ (surjective) topological isomorphism or $G-b(G)$ generates $G$.

Proof. By Lemma $1.1, b(G)$ is a closed subset of $G$ and $b: G \rightarrow b(G)$ is a topological isomorphism. If $G-b(G) \neq \varnothing$, then the subgroup of $G$ generated by $G-b(G)$ is clopen, since $G-b(G)$ is open, which forces $G-b(G)$ to generate $G$, since $G$ is connected. 
Proposition 3.3. Let $J \subset E^{1}$ and $S=J \times E^{1}$. Let $f: S \rightarrow E^{1}$ be a continuous function such that $0<m \leq(f(x, y)-f(x, z)) /(y-z)$ for some $m$, each $x \in J$ and for any distinct $y, z \in E^{1}$. Then there exists a unique continuous function $g: J \rightarrow E^{1}$ such that $f(x, g(x))=0$ for all $x \in J$.

Proof. For each $x \in J$, let $b_{x}: E^{1} \rightarrow E^{1}$ be defined by $b_{x}(y)=f(x, y)$. Clearly $b_{x}$ is a continuous m-enlarger, for each $x \in J$. Therefore, by Theorem 2.2, there exists (a unique) $y_{x} \in E^{1}$ such that $0=b_{x}\left(y_{x}\right)=f\left(x, y_{x}\right)$, for each $x \in J$.

Now, we let $g: J \rightarrow E^{1}$ be defined by $g(x)=y_{x}$, for each $x \in J$. It remains to check that $g$ is continuous. Suppose $g$ is not continuous at $x$. Then there exists $\epsilon>0$ and sequence $\left\{x_{n}\right\}$ such that $\lim _{n} x_{n}=x$ and $\left|g\left(x_{n}\right)-g(x)\right| \geq \epsilon$ for $n=1,2, \ldots$. From the continuity of $f$ and $f(x, g(x))=$ 0 , there exists an integer $n_{0}$ such that $f\left(x_{n}, g(x)\right)<m \epsilon$ for $n \geq n_{0}$. On the other hand, from the condition satisfied by $f$, one gets that

$$
\left|f\left(x_{n}, g(x)\right)\right|=\left|f\left(x_{n}, g\left(x_{n}\right)\right)-f\left(x_{n}, g(x)\right)\right| \geq m\left|g\left(x_{n}\right)-g(x)\right| \geq m \epsilon
$$

for $n \geq n_{0}$. We have a contradiction, which completes the proof.

The technique just completed is quite general and can clearly be applied to the following result and many others like it.

Proposition 3.4. Let $f: E^{n} \rightarrow E^{1}$ be a continuous function such that

$$
0<m \leq \frac{f\left(x_{1}, \cdots, x_{i}, \cdots, x_{n}\right)-f\left(x_{1}, \cdots, y_{i}, \cdots, x_{n}\right)}{x_{i}-y_{i}}
$$

for some $1 \leq i \leq n$, some $m$ and distinct $x_{i}, y_{i}$. Then there exists a unique continuous function $g_{i}: E^{n-1} \rightarrow E^{1}$ such that

$$
f\left(x_{1}, \cdots, x_{i-1}, g_{i}(\bar{x}), x_{i+1}, \cdots, x_{n-1}\right)=0
$$

for all $\bar{x}=\left(x_{1}, \ldots, x_{n-1}\right) \in E^{n-1}$.

Proposition 3.5. Let $S$ be an open subset of $E^{n},\left(p_{1}, \ldots, p_{n}\right)=$ $\bar{p} \in S, f: S \rightarrow E^{1}$ a continuous function sucb that $f(\bar{p})=0$ and (1) bolds for some $1 \leq i \leq n$, some $m$ and distinct $x_{i}, y_{i}$. Then there exists a neighborbood $W$ of $\left(p_{1}, \ldots, p_{i-1}, p_{i+1}, \ldots, p_{n}\right)$ and a unique continuous function $g_{i}: W \rightarrow E^{1}$ such that (2) bolds for all $\bar{x}=\left(x_{1}, \ldots, x_{n-1}\right) \in W$.

Proof. Let $l=\left\{p_{1}\right\} \times \cdots \times\left[p_{i}-\epsilon, p_{i}+\epsilon\right] \times \cdots \times\left\{p_{n}\right\} \subset S$ and $f_{i}=$ $f \mid l$. From hypothesis, $f_{i}$ is one-to-one and

$$
f_{i}\left(p_{1}, \cdots, p_{i}+\epsilon, \cdots, p_{n}\right)>0, \quad f_{i}\left(p_{1}, \cdots, p_{i}-\epsilon, \cdots, p_{n}\right)<0 .
$$


Using the local compactness of $S$, it is then easy to find an $n$-cube

$$
T=\left[p_{1}-\delta, p_{1}+\delta\right] \times \cdots \times\left[p_{i}-\epsilon, p_{i}+\epsilon\right] \times \cdots \times\left[p_{n}-\delta, p_{n}+\delta\right] \subset S
$$

such that

$$
f_{i}\left(x_{1}, \cdots, p_{i}+\epsilon, \cdots, x_{n}\right)>0, \quad f_{i}\left(x_{1}, \cdots, p_{i}-\epsilon, \cdots, x_{n}\right)<0,
$$

for each $\left(x_{1}, \ldots, x_{i-1}, x_{i+1}, \ldots, x_{n}\right) \in W$, where $W$ is the $(n-1)$-cube obtained from $T$ by removing its $i$-factor. Essentially using the techniques of the proof of Proposition 3.3, it is now easy to construct a function $g_{i}: W \rightarrow E^{1}$ with the required properties, which concludes the proof.

It is noteworthy that the preceding result applies to functions $f$ whose partial derivative $\partial f / \partial x_{i}$ is continuous on a neighborhood of $\bar{p}$ and $\partial f(\bar{p}) / \partial x_{i} \neq 0$ (if $\partial f(\bar{p}) / \partial x_{i}<0$, we consider the function $-f$ ).

4. Conclusion. In recent years, the theory of fixed points of nonexpansive maps has been intensively studied. Various significant results have been proved, which complement the Banach contraction principle.

However, no real attention has been given to expansions or enlargers. We hope that we have demonstrated that these concepts are indeed very useful in spaces with the Brouwer property. (An extensive study of these spaces, by E. Duda, appears in Duke Math. J. 30 (1963), 647-660.)

DEPARTMENT OF MATHEMATICS, UNIVERSITY OF CALIFORNIA, DAVIS, CALIFORNIA 95616 